\title{
Spatially Enabled COVID-19: A Review of Applications and Systems
}

\author{
Abbas Rajabifard, Yiqun Chen, Yibo Zhang and Katie Potts
}

The ongoing COVID-19 pandemic has profoundly reshaped the world and impacted the lives of billions globally across many facets, including health, economy, culture, education, environment and politics. Since declared as a public health emergency of international concern (PHEIC) in January 2020, the outbreak has attracted significant research attention globally. Governments, industries and academics altogether are investigating various means for monitoring the spread of the virus, assessing the impacts, and planning strategies and policies for reopening. Many tools and applications have been developed to support government and emergency agencies for critical decision-making and situation monitoring at various stage of the outbreak. This paper reviews existing COVID-19 emergency management tools and applications currently being adopted by different countries and jurisdictions, and identifies and compares their key capabilities and functionalities.

\subsection{Introduction}

The outbreak of the COVID-19 virus has fundamentally changed the way our world operates. The impact of this virus has been felt in almost every country around the globe, disrupting and putting extreme pressure on various industry sectors - such as building and construction, retail, transport, hospitality, education, financial services, agriculture, aviation and tourism, and healthcare. In response to the pandemic, the governments of many countries have adopted strategies to minimise the spread and impact of the virus, which involves quarantining communities through lockdown mandates which have had enormous economic and social impacts. As the spread of infectious disease is inherently a spatial process, the geospatial industry which specialises in geospatial data, technologies, and analytical methods play a critical role in understanding and responding to the coronavirus disease 2019 (COVID-19) pandemic [1]. To contribute and assist with the escalating problem, the geospatial industry, like many other industries, have focused their attention to this global challenge and have founded solutions and developed tools which aid and assist officials in their role of managing this unprecedented event.

Areas of focus which fit within the scope of the geospatial industry's expertise include: developing spatial data infrastructures (SDI) for surveillance and data sharing; incorporating mobility data into infectious disease forecasting; using geospatial technologies for digital contact tracing; integrating geographic data in COVID-19 modelling; investigating social vulnerabilities and health disparities; communicating the status of the disease or status of facilities for return-to-normal operations; and tracking, monitoring and optimisation of the location of necessary health and safety resources such as personal protective equipment (PPE), ventilators, and available hospital beds [1]. 
One early contribution from the geospatial industry has been the development of smartphone apps to assist contact tracing. Contact tracing involves identifying persons who may have come into contact with an infected person, and the management these people who have been exposed to COVID-19 to prevent onward transmission [2]. It is an essential activity conducted by public health organisations for controlling the virus. Through tracing of the contacts of infected individuals, testing them for infections, and then isolating the infected, public health aims to reduce the infections in the population. In order to effectively perform contact tracing, specific location and time details are required. To assist with this task, the geospatial industry has responded with a range of applications which can facilitate the tracing, some assisting from the public health side, and others from the citizen side. The apps take advantage of the inbuilt location and timestamp records of smart phones, GPS data from cars, credit card transactions, travel histories and CCTV footage to determine where and at what time a person has been in a specific location, and through analysis can use this information to determine intersections of individuals to determine who has been potentially exposed. Identifying the exact location of sick people, tracing their movements, and isolating them minimises the need to impose mobility restrictions or business closures [3].

Another application which has seen geospatial technology play a critical role has been in the identification and proximities of individuals to essential services. The geospatial industry has developed smartphone applications for use by citizens and also complex platforms to perform detailed analysis for government health departments. Government, industry and academia have put significant efforts and resources to build these applications, which fall into two categories of tracing apps and map-enabled dashboards. These applications are all built upon the same COVID-19 test data, and utilise various technologies and aggregate additional data sources to serve their purposes.

\subsection{Tracing Apps}

Tracing apps are critical means for the pandemic control, particularly when widely deployed, as they help to detect and notify people who contact with a carrier and can also monitor who breach the isolation rules. In March 2020, Ferretti et al. [4] suggested using digital contact tracing to quantify the virus transmission and proposed a schematic of a tracing app (Figure 16.1 (a)). Contacts of carrier A are traced by the app, and when carrier A is confirmed by a positive test result, the app triggers an instant notification to all the contacts with risk-stratified quarantine (i.e., close contacts B, C, D, E, F, G and low-risk contacts H, I) and physical distancing advice. CDC also released a COVID-19 contact tracing workflow (Figure 16.1 (b)) which can help scientists follow the chain of infection to understand how the virus transmits among crowd [5].

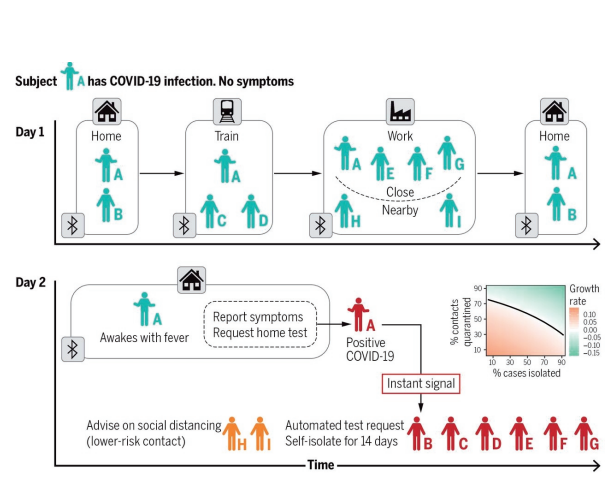

(a)

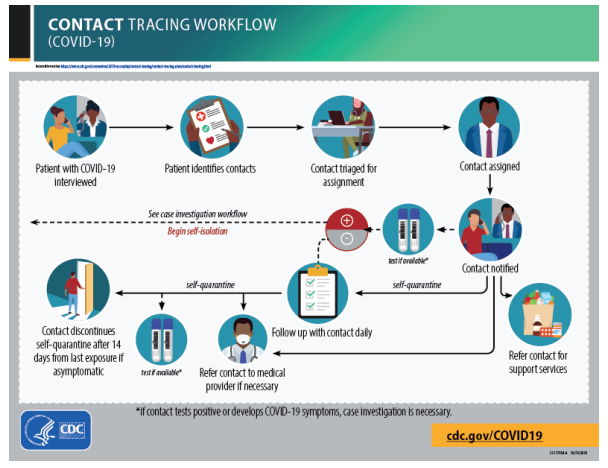

(b)

\section{FIGURE 16.1}

a) A schematic of a tracing app, b) COVID-19 contact tracing workflow 
Most of the apps adopt Bluetooth technology to detect the proximity to other mobile devices. Unlike GPS or Wi-Fi data, the Bluetooth technology only tracks which device has been near one another, rather than reporting users' actual locations. They are usually classified as "Decentralised Contact Tracing" and considered to be better privacy-preserving and less intrusive, comparing with "Centralised Contact Tracing" methods which utilise cellular network and GPS to determine the location of users. In March 2020, in collaboration with CoEPi [6], Covid Watch [7] was the first team in the world to develop an open-source, anonymous, decentralised Bluetooth digital contact tracing protocol, the CEN (Contact Event Numbers) Protocol. It now has been renamed as TCN (Temporary Contact Numbers) Protocol[8]. In April 2020, similar decentralised protocols like DP-3T (Decentralized Privacy-Preserving Proximity Tracing) [8], PACT(Private Automated Contact Tracing) [9] and Google/Apple Exposure Notification framework [10] were also prevailing. By adopting such a decentralised protocol, contact tracing apps will create and broadcast short-lived pseudorandom values over Bluetooth. These values are recorded by nearby devices and reveal no information about users' identity or location history as they are pseudorandom. When a user is developing symptoms or tested positive, the app will send a report to any potential contacts by uploading a packet of data to a server. Other users can monitor data published by the server to learn whether they have received any reports $[11,12]$.

Several issues impact the effectiveness of tracking apps in the real world. First, inferring physical distance based these technologies can be unreliable. The range $(3-10 \mathrm{~m})$ of Bluetooth-enabled device varies dramatically due to the environment or the way the device is held $[13,14]$. GPS-based proximity detection can also be unreliable, smartphones are typically accurate to within a 4.9-meter radius in open space, with accuracy decreasing further in the presence of signal blockage $[15,16]$. The cellular network-based proximity reasoning also depends on the density of antenna towers (base stations) and the precision of positioning can achieve down to 50 meters in urban areas [17]. While, in most countries, the social distancing guidelines recommend 1.5 to 2 meters, which could not be reliably and accurately detected by any of these means. False positives might lead to unnecessary self-quarantine and could cause the public to ignore warnings when they find these warnings are untrustworthy. Another problem is the update ratio of tracing apps. The effectiveness of a tracing app depends on how many people use it regularly. If only a small proportion of people participate in, the app is worthless and could be harmful as its indications will be highly inaccurate and could even instill a false sense of security [18]. In Singapore, by the end of September 2020, 2.4 million people ( $41 \%$ population) downloaded the TraceTogether App. The uptake was lower than the optimal number of users required for the contact tracing system to work well, which was $75 \%$ of the population [19].

Australia launched its contact tracking app CovidSafe in April, which is completely voluntary. By the end of September, it has accumulated over 7 million downloads ( $28 \%$ population), while how and whether the app is being used has yet been revealed $[20,21]$. In the UK, the second version of the NHS Covid-19 app has over 10 million downloads (around $15 \%$ population) since it launched in September 2020 [13, 22-24]. As the tracing apps are characterised by strong network effect [18], its efficacy is the square of the proportion of the population using the app, multiplied by the probability of the app detecting infectious contacts, multiplied by the fractional reduction in infectiousness resulting from being notified as a contact [4]. Simulation model shows that approximately $60 \%$ of the whole population are required to use the app and adhere to the app's recommendations to stop virus contagion [25].

\subsection{Map-Based Dashboard}

The history of using maps to understand the spread of disease can go back to 1850's when Dr. John Snow connected location and illness to trace the source of a cholera outbreak in London, as shown in Figure 16.2 (a) [26]. From disease atlases in early 20th century to more recent web mapping of Ebola (Figure 16.2 (b) [27]) and Zika, maps have been considered as a critical tool in coping with contagious viruses [28]. The reason behind is that global mobility is faster and easier than ever 
before, and a carrier can become a super spreader, infecting a large number of people across a large geographic area [28].

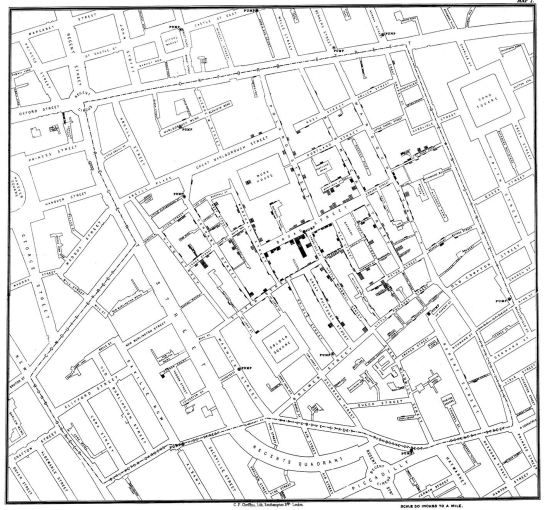

(a)

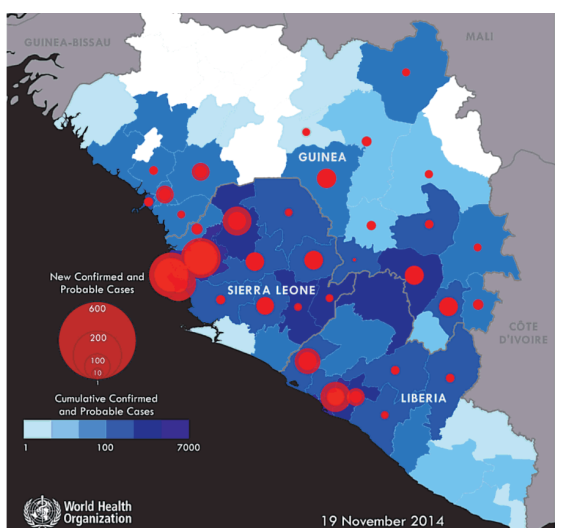

(b)

\section{FIGURE 16.2}

a) Cluster map of cholera cases in London, 1854, b) Web mapping of Ebola virus in Africa, 2014

The most famous COVID-19 dashboard (see Figure 16.3) has been created and maintained by Johns Hopkins University since late January 2020. It is a map-based web application, aggregating real-time information about the pandemic at the global level (190 countries and regions included), and has been cited as official COVID-19 data and statistics by many media channels. The dashboard sources and aggregates data globally and reports cases at the province level in China; at the city level in the USA, Australia, and Canada; and at the country level otherwise [29, 30]. The interactive maps of the dashboard include accumulated cases, active cases, incidence rate, case-fatality rate and testing rate. Besides, it also offers critical trends analysis and interactive visualisation, which help user unfold details about the outbreak spread patterns at various geographical levels [31-33].

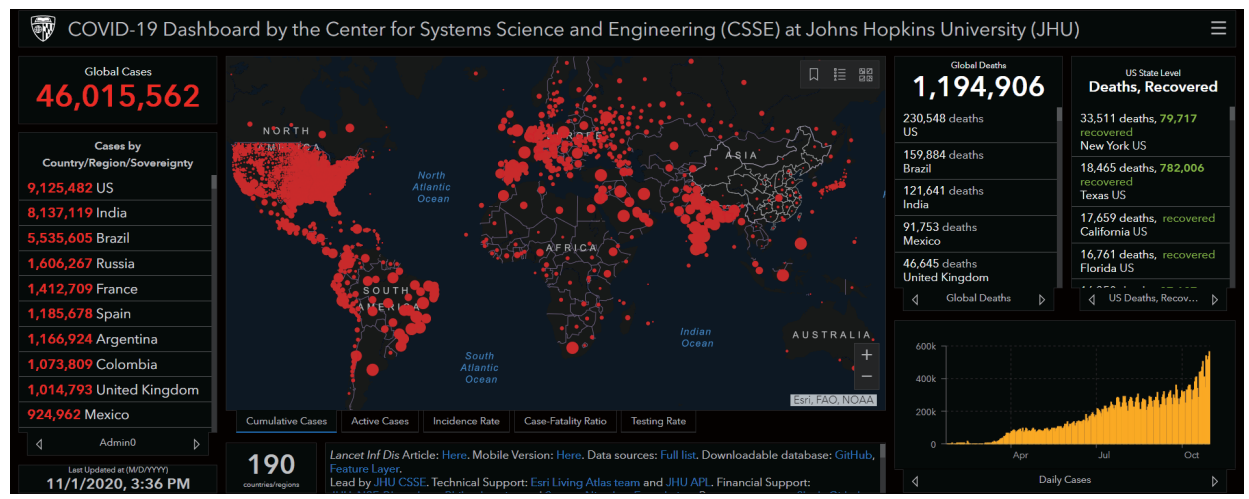

\section{FIGURE 16.3}

COVID-19 Dashboard developed by Johns Hopkins University

The Johns Hopkins COVID-19 dashboard has a significant impact on the coming COVID-19 dashboard design and functionalities. Within a short period, many countries and organisations have adopted similar web mapping technology (mostly powered by ESRI Live Atlas) to build their dashboard and customise with additional information [28]. Figure 16.4 shows four dashboards for Italy (top-left), China (top-right), Japan (bottom-left) and Germany (bottom-right) respectively [34-37]. They are all built upon ESRI ArcGIS online map applications and share the same style with 
Johns Hopkins University's COVID-19 dashboard. Figure 16.5 illustrates the COVID-19 dashboards created by India (top-left), Brazil (top-right), Australia (bottom-left) and WHO (bottom-right) using different web tools. Though the appearances are different from each other, the maps and statistics charts all remain as the key components [38-41].
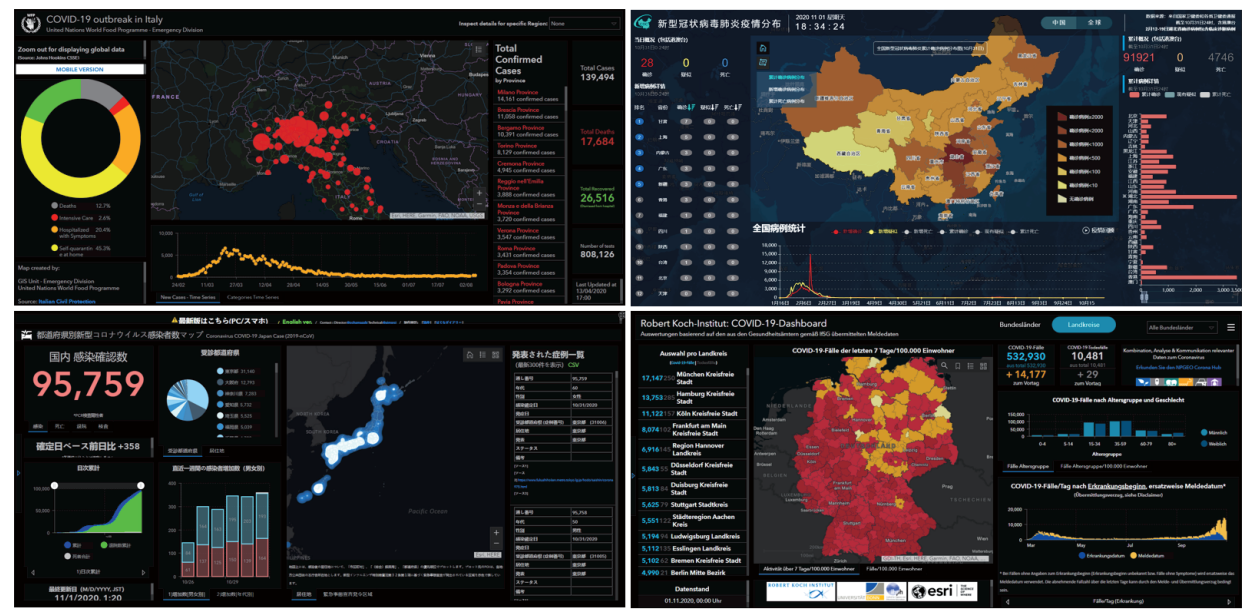

FIGURE 16.4

A series of similar COVID-19 dashboards created with ESRI ArcGIS Online
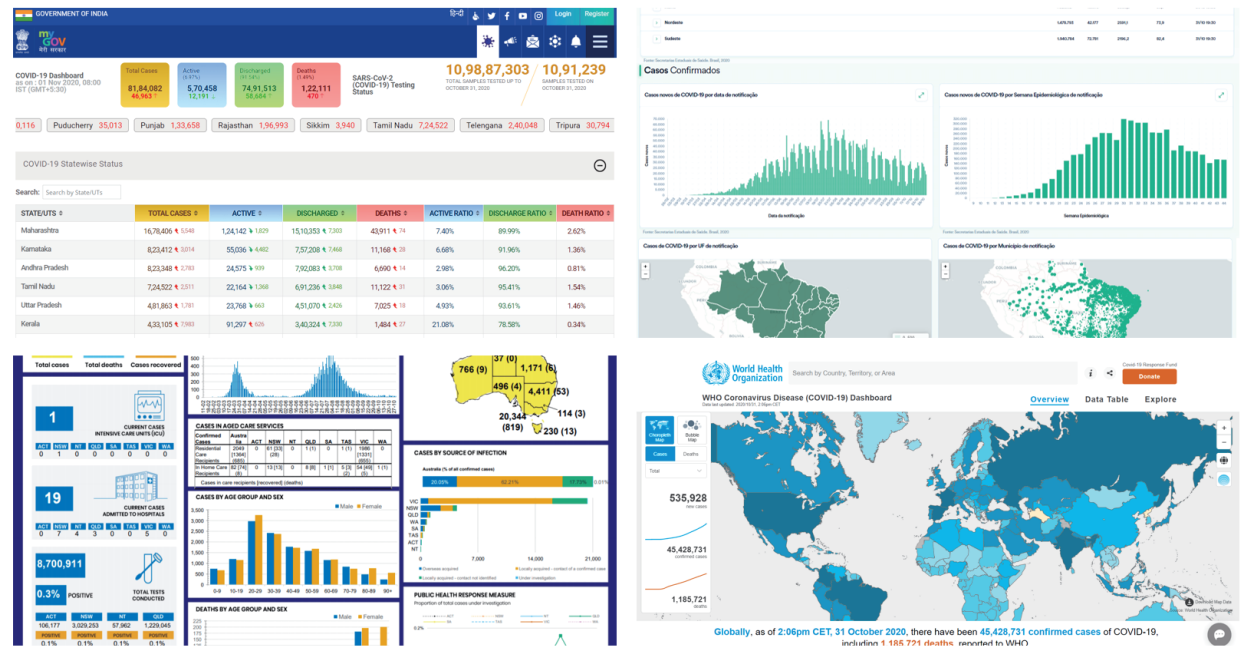

\section{FIGURE 16.5}

COVID-19 Dashboards created using different web tools

Besides diseases statistics, dispersion maps and propagation animations, there is another category of dashboards particularly focus on the pandemic impact analyses including mobility, health, economy and society. For example, the COVID-19 Impact Analysis Platform [42, 43] developed by the University of Maryland placed emphases on the mobility and social impact of the virus by incorporating over 30 variables, including social distancing index (top-left), percentage of hospital bed utilisation (top-right), unemployment rate (bottom-left) and COVID death rate (bottom-right), as shown in Figure 16.6. 

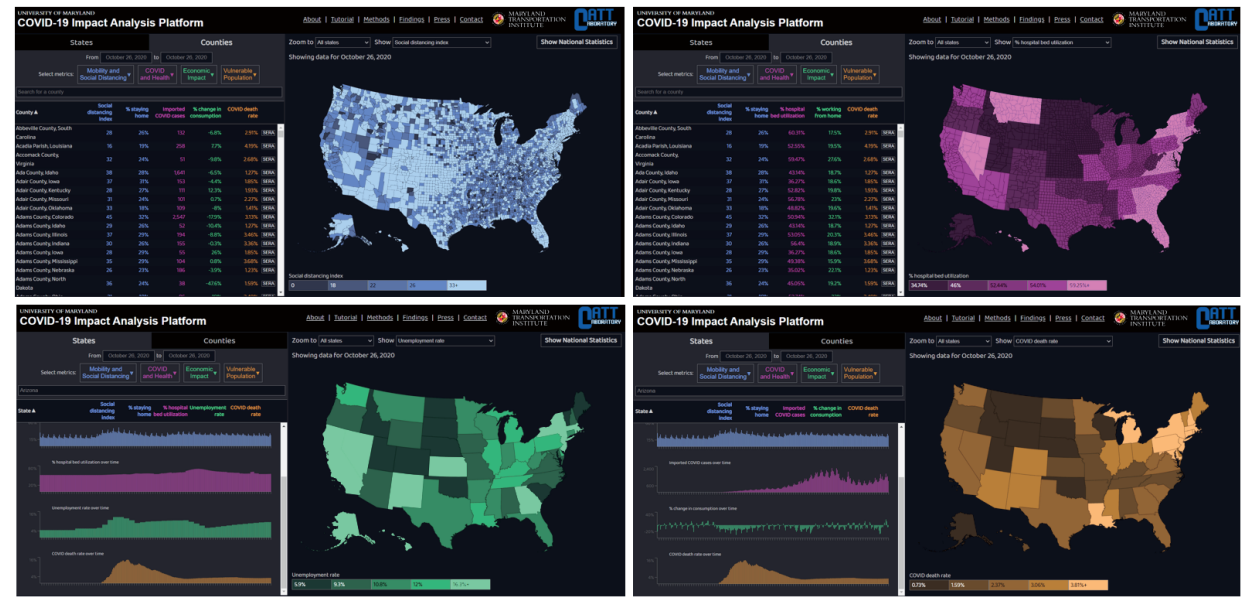

\section{FIGURE 16.6}

COVID-19 Impact Analysis Platform developed by University of Maryland

The Australia COVID-19 Location Tracker developed by the Centre for Disaster Management and Public Safety (CDMPS) jointly with the Centre for SDIs and Land Administration (CSDILA), both at the University of Melbourne also comes with a set of ready-for-use analytics tools by utilising live data through multiple sources for Australia.

The system can perform capacity and service area (e.g., 3, 5, $10 \mathrm{~km}$ ) analysis for COVID-19 related hospitals and clinics and provide insights medical resource supply chain management at various scenarios. It plots the distribution of the vulnerable population (e.g., $65+$ years old) at a fine geospatial unit level based on the latest Census data and identifies regions required particular attention by cross-referencing health condition datasets. The system compiles and visualises the number of closed non-essential businesses during various stage of lockdown, and estimates the impacts on the local economy such as unemployment rate and scale of subsidies etc.
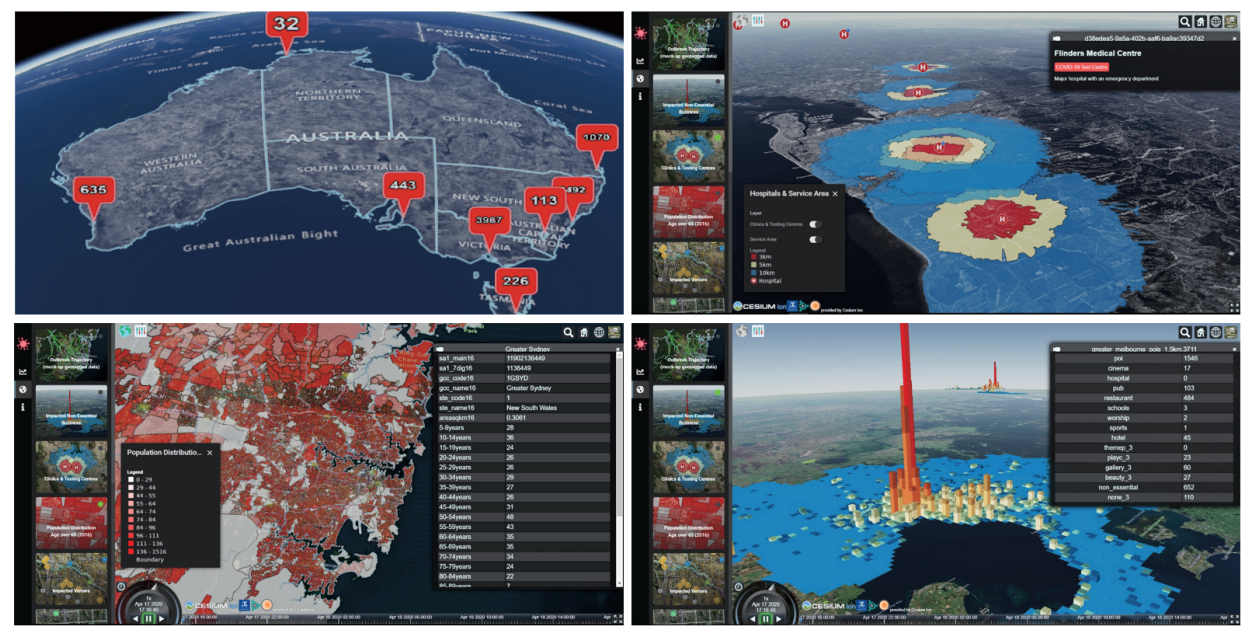

\section{FIGURE 16.7}

Australia COVID-19 Location Tracker developed by the University of Melbourne

In general, the map-based dashboards prevail in the COVID-19 pandemic and have been adopted worldwide at various administrative scales. By aggregating, analysing and conveying data in a timely manner, it serves as a critical and effective tool for government and public to communicate 
and understand the spread of disease and hence helps to increase the pandemic situational awareness and preparedness.

\subsection{Conclusion}

The geospatial community has a fundamental and critical role to play in supporting government management of COVID-19 through contact tracing and map development applications. In this paper tracing apps that have been developed have been presented and discussed revealing their strengths and weaknesses as well as challenges faced when trying to successfully implement these apps at a large scale to produce valuable data for management of the virus. Successful map dashboards that are serving both government and the community were also presented and discussed. Consensus on these applications are that map dashboards have been the most useful and more widely accepted and adopted, proving an effective tool for presenting and relaying information for government and public understanding and situational awareness on COVID-19.

\section{References}

[1] Charlotte D. Smith and J. M. Incorporating Geographic Information Science and Technology in Response to the COVID-19 Pandemic. 2020. URL https://www.cdc.gov/pcd/issues/2020/20_0246.htm. Accessed on 10 October 2020 .

[2] WHO. Rolling updates on coronavirus disease (COVID-19). 2020. URL https://bit.ly/39fHuz8. Accessed on 10 October 2020.

[3] Mika-Petteri Torhonen, S.E., Dongkyu Kwak Antos, and Alvaro Federico Barra. The role of geospatial information in confronting COVID-19 - Learning from Korea. 2020. URL https://bit.1y/33iQGPo. Accessed on 1 August 2020.

[4] L. Ferretti and et al. Quantifying SARS-CoV-2 transmission suggests epidemic control with digital contact tracing. Science, 368(6491): eabb6936, 2020.

[5] CDC. Monitoring and tracking the disease. 2020. URL https://bit.ly/3fwD6g4. Accessed on 21 October 2020 .

[6] CoEpi. Monitoring and tracking the diseaseCoEpi: Community Epidemiology in Action. 2020. URL https: //www. coepi.org/. Accessed on 10 October 2020.

[7] Covid Watch. 2020. URL https://www.covidwatch.org/. Accessed on 10 October 2020.

[8] DP-3T. Decentralised Privacy-Preserving Proximity Tracing. 2020. URL https://github.com/DP-3T/ documents. Accessed on 10 October 2020.

[9] MIT. PACT: Private Automated Contact Tracing. 2020. Accessed on 10 October 2020.

[10] Google. Exposure Notifications: Using technology to help public health authorities fight COVID-19. 2020. URL https://www.google.com/covid19/exposurenotifications/. Accessed on 29 August 2020.

[11] T. Coalition. TCN Protocol. 2020. URL https://github.com/TCNCoalition/TCN. Accessed on 10 October 2020.

[12] HD VALENCE. Private Contact Tracing Protocols Compared: DP-3T and CEN. 2020. URL https://bit. ly/2KvLmRY. Accessed on 15 Septermber 2020.

[13] D Leprince-Ringuet. Contact-tracing apps: Why the NHS said no to Apple and Google's plan. 2020. URL https://zd.net/39cjblk. Accessed on 29 August 2020.

[14] J. Larsson. Distance estimation and positioning based on Bluetooth low energy technology. KTH Royal Institute of Technology, 2015.

[15] Frank van Diggelen and P.E. The World's first GPS MOOC and Worldwide Laboratory using Smartphones. in Proceedings of the 28th International Technical Meeting of the Satellite Division of The Institute of Navigation (ION GNSS+ 2015). Tampa, Florida, 2015. 
[16] GPS.GOV. How accurate is GPS? 2020. URL https://ww.gps.gov/systems/gps/performance/accuracy/. Accessed on 29 August 2020.

[17] H. Laitinen, J. Lahteenmaki, and T. Nordstrom. Database correlation method for GSM location. IEEE.

[18] Chiara Farronato, M.I., Marcin Bartosiak, Stefano Denicolai, Luca Ferretti, and Roberto Fontana. How to Get People to Actually Use Contact-Tracing Apps. 2020. URL https://hbr.org/2020/07/how-to-get-peopleto-actually-use-contact-tracing-apps. Accessed on 1 September 2020.

[19] H. Baharudin. Contact-tracing device will not track location; people can use TraceTogether if they prefer, says Vivian Balakrishnan. 2020. URL https://bit.ly/33esKfX. Accessed on 29 August 2020.

[20] A. COVIDSafe app Department of Health. he COVIDSafe app is a tool that helps identify people exposed to coronavirus (COVID-19). 2020. URL https://www.health.gov.au/resources/apps-and-tools/covidsafe-app. Accessed on 10 September 2020.

[21] T. Guardian. Releasing Covidsafe app usage numbers could risk public safety, government claims. 2020. URL https://bit.ly/33fqxka. Accessed on 1 October 2020.

[22] Matt Burgess. Everything you need to know about the new NHS contact tracing app. 2020. URL https: //bit.1y/3nUG01g. Accessed on 25 October 2020.

[23] Department of Health and Social Care. NHS COVID-19 app has been downloaded over 10 million times. 2020. URL https://bit.1y/2UZj707. Accessed on 22 October 2020.

[24] Joe O'Halloran. NHS Covid-19 app exceeds 10 million downloads, but has teething troubles. 2020. URL https://bit.1y/313L69x. Accessed on 25 October 2020.

[25] Oxford University. Digital contact tracing can slow or even stop coronavirus transmission and ease us out of lockdown. 2020. URL https://bit.1y/378Gz0u. Accessed on 21 October 2020.

[26] Wikipedia. John Snow. 2020. URL https://en.wikipedia.org/wiki/John_Snow. Accessed on 28 August 2020.

[27] WHO. Ebola maps. 2016. URL https://www.who.int/csr/disease/ebola/maps/en/. Accessed on 20 October 2020 .

[28] E. Geraghty. Coronavirus: World Connectivity Can Save Lives. 2020. URL https://bit.ly/3152bQv. Accessed on 29 August 2020.

[29] E. Dong, H. Du, and L. Gardner. An interactive web-based dashboard to track COVID-19 in real time. The Lancet Infectious Diseases. 20(5) 533-534, 2020.

[30] Johns Hopkins University. COVID-19 Data Repository by the Center for Systems Science and Engineering (CSSE) at Johns Hopkins University. 2020. URL https://github.com/CSSEGISandData/COVID-19. Accessed on 29 October 2020.

[31] Johns Hopkins University. Animated Maps. 2020. URL https://coronavirus.jhu.edu/data/animated-worldmap. Accessed on 1 November 2020.

[32] Johns Hopkins University. Daily Confirmed New Cases (7-Day Moving Average). 2020. URL https:// coronavirus.jhu.edu/data/new-cases. Accessed on 1 November 2020.

[33] Johns Hopkins University. Cases and mortality by country. 2020. URL https://coronavirus.jhu.edu/data/ mortality. Accessed on 1 November 2020.

[34] UNWFP. COVID-19 outbreak in Italy. 2020. URL https://bit.ly/2KDLG1g. Accessed on 1 November 2020.

[35] C. CHINA. 2020. URL http://2019ncov.chinacdc.cn/2019-nCoV/. Accessed on 1 November 2020.

[36] J.A.G JAPAN Corp. Coronavirus COVID-19 Japan Case by Each Prefecture. 2020. URL https://bit.1y/ 3nZguId. Accessed on 1 November 2020.

[37] R. Koch-Institut. COVID-19 Dashboard. 2020. URL https://experience.arcgis.com/experience/ 478220a4c454480e823b17327b2bf1d4. Accessed on 1 November 2020.

[38] Government of India. COVID-19 Dashboard. 2020. URL https://www.mygov.in/covid-19/. Accessed on 1 November 2020 .

[39] M.d.S. Brazil. COVID19 Painel Coronavírus. 2020. URL https://covid.saude.gov.br/. Accessed on 1 November 2020

[40] Australian Government Department of Health. Coronavirus (COVID-19) at a glance. 2020. URL https: //bit.ly/3kZRnTL. Accessed on 1 November 2020.

[41] WHO. WHO Coronavirus Disease (COVID-19) Dashboard. 2020. URL https://covid19.who.int. Accessed on 1 November 2020.

[42] University of Maryland. COVID-19 Impact Analysis Platform. 2020. URL https://data.covid.umd.edu/ about/index.html.

[43] Lei Zhang, Sepehr Ghader, Michael L Pack, Chenfeng Xiong, Aref Darzi, Mofeng Yang, Qianqian Sun, AliAkbar Kabiri, and Songhua Hu. An Interactive COVID-19 Mobility Impact and Social Distancing Analysis Platform. medRxiv, 2020. doi: 10.1101/2020.04.29.20085472. 